\title{
Clark-Ocone Formula for Generalized Functionals of Discrete-Time Normal Noises
}

\author{
Caishi Wang $(\mathbb{D}$, Shuai Lin $(\mathbb{D}$, and Ailing Huang $\mathbb{D}$ \\ School of Mathematics and Statistics, Northwest Normal University, Lanzhou, Gansu 730070, China \\ Correspondence should be addressed to Caishi Wang; wangcs@nwnu.edu.cn
}

Received 28 November 2017; Accepted 4 January 2018; Published 6 February 2018

Academic Editor: Pasquale Vetro

Copyright (C) 2018 Caishi Wang et al. This is an open access article distributed under the Creative Commons Attribution License, which permits unrestricted use, distribution, and reproduction in any medium, provided the original work is properly cited.

The Clark-Ocone formula in the theory of discrete-time chaotic calculus holds only for square integrable functionals of discretetime normal noises. In this paper, we aim at extending this formula to generalized functionals of discrete-time normal noises. Let $Z$ be a discrete-time normal noise that has the chaotic representation property. We first prove a result concerning the regularity of generalized functionals of $Z$. Then, we use the Fock transform to define some fundamental operators on generalized functionals of $Z$ and apply the abovementioned regularity result to prove the continuity of these operators. Finally, we establish the Clark-Ocone formula for generalized functionals of $Z$ and show its application results, which include the covariant identity result and the variant upper bound result for generalized functionals of $Z$.

\section{Introduction}

One of the important theorems in Privault's discrete-time chaotic calculus $[1,2]$ is its Clark-Ocone formula, which reads

$$
\xi=\mathbb{E} \xi+\sum_{k=0}^{\infty} Z_{k} \mathbb{E}\left[\partial_{k} \xi \mid \mathscr{F}_{k-1}\right], \quad \xi \in \mathscr{L}^{2}(Z)
$$

where $Z=\left(Z_{k}\right)$ is a discrete-time normal noise, $\mathscr{L}^{2}(Z)$ is the space of square integrable functionals of $Z, \mathscr{F}_{k}$ is the $\sigma$-field generated by $\left(Z_{j} ; 0 \leq j \leq k\right), \partial_{k}$ is the annihilation operator on $\mathscr{L}^{2}(Z)$, and the series on the right-hand side converges in the norm of $\mathscr{L}^{2}(Z)$.

The Clark-Ocone formula (1) directly gives the predictable representation of functionals of $Z$, which implies the predictable representation property of discrete-time martingales associated with $Z$. The formula can also be used to establish the corresponding covariant identities [1]. More importantly, as was shown by Gao and Privault [3], this formula plays an important role in proving logarithmic Sobolev inequalities for Bernoulli measures. There are other applications based on the formula [2].

Despite its multiple uses, however, the Clark-Ocone formula (1) still suffers from a main drawback. That is, it holds only for the square integrable functionals $\xi$ of $Z$, which excludes many other interesting functionals of $Z$.

On the other hand, as is shown in [4], one can use the canonical orthonormal basis of $\mathscr{L}^{2}(Z)$ to construct a nuclear space $\mathcal{S}(Z)$ such that $\mathcal{S}(Z)$ is densely contained in $\mathscr{L}^{2}(Z)$. Thus, by identifying $\mathscr{L}^{2}(Z)$ with its dual, one can get a Gel'fand triple

$$
\mathcal{S}(Z) \subset \mathscr{L}^{2}(Z) \subset \mathcal{S}^{*}(Z),
$$

where $\delta^{*}(Z)$ is the dual of $\delta(Z)$, which is endowed with the strong topology, which cannot be induced by any norm [5]. As usual, $\delta(Z)$ is called the testing functional space of $Z$, while $\delta^{*}(Z)$ is called the generalized functional space of $Z$. It turns out [6] that the generalized functional space $\mathcal{S}^{*}(Z)$ can accommodate many quantities of theoretical interest that cannot be covered by $\mathscr{L}^{2}(Z)$.

In this paper, we would like to extend the Clark-Ocone formula (1) to the generalized functionals of $Z$. More precisely, we would like to establish a Clark-Ocone formula for all elements of $\mathcal{S}^{*}(Z)$. Our main work is as follows.

We first prove a result concerning the regularity of generalized functionals in $\mathcal{S}^{*}(Z)$ in Section 2. Then, in Section 3, we use the Fock transform [6] to define some fundamental operators on $\mathcal{S}^{*}(Z)$ and apply the abovementioned 
regularity result to prove the continuity of these operators. Finally, we establish our formula, namely, the Clark-Ocone formula, for generalized functionals in $\mathcal{S}^{*}(Z)$ in Section 3 and show its application results in Section 4 , which include the covariant identity result and the variant upper bound result for generalized functionals in $\delta^{*}(Z)$.

Throughout this paper, $\mathbb{N}$ designates the set of all nonnegative integers and $\Gamma$ the finite power set of $\mathbb{N}$; namely,

$$
\Gamma=\{\sigma \mid \sigma \subset \mathbb{N}, \#(\sigma)<\infty\},
$$

where $\#(\sigma)$ means the cardinality of $\sigma$ as a set. If $k \in \mathbb{N}$ and $\sigma \in \Gamma$, then we simply write $\sigma \cup k$ for $\sigma \cup\{k\}$. Similarly, we use $\sigma \backslash k$.

\section{Generalized Functionals of Discrete-Time Normal Noises}

In all the following sections, we always assume that $(\Omega, \mathscr{F}, P)$ is a given probability space. We use $\mathbb{E}$ to mean the expectation with respect to $P$. As usual, $\mathscr{L}^{2}(\Omega, \mathscr{F}, P)$ denotes the Hilbert space of square integrable complex-valued measurable functions on $(\Omega, \mathscr{F}, P)$. We use $\langle\cdot, \cdot\rangle$ and $\|\cdot\|$ to mean the inner product and norm of $\mathscr{L}^{2}(\Omega, \mathscr{F}, P)$, respectively. By convention, $\langle\cdot, \cdot\rangle$ is conjugate-linear in its first argument and linear in its second argument.

2.1. Discrete-Time Normal Noises. A sequence $Z=\left(Z_{n}\right)_{n \in \mathbb{N}}$ of integrable random variables on $(\Omega, \mathscr{F}, P)$ is called a discretetime normal noise if it satisfies

(i) $\mathbb{E}\left[Z_{n} \mid \mathscr{F}_{n-1}\right]=0$ for $n \geq 0$;

(ii) $\mathbb{E}\left[Z_{n}^{2} \mid \mathscr{F}_{n-1}\right]=1$ for $n \geq 0$.

Here, $\mathscr{F}_{-1}=\{\emptyset, \Omega\}, \mathscr{F}_{n}=\sigma\left(Z_{k} ; 0 \leq k \leq n\right)$ for $n \in \mathbb{N}$ and $\mathbb{E}\left[\cdot \mid \mathscr{F}_{n}\right]$ means the conditional expectation given $\mathscr{F}_{n}$.

Example 1. Let $\zeta=\left(\zeta_{n}\right)_{n \in \mathbb{N}}$ be an independent sequence of random variables on $(\Omega, \mathscr{F}, P)$ with

$$
P\left\{\zeta_{n}=-1\right\}=P\left\{\zeta_{n}=1\right\}=\frac{1}{2}, \quad n \in \mathbb{N} .
$$

Write $\mathscr{G}_{-1}=\{\emptyset, \Omega\}$ and $\mathscr{G}_{n}=\sigma\left(\zeta_{k} ; 0 \leq k \leq n\right)$ for $n \in \mathbb{N}$. Then, one can immediately see that

(i) $\mathbb{E}\left[\zeta_{n} \mid \mathscr{G}_{n-1}\right]=0$ for $n \geq 0$;

(ii) $\mathbb{E}\left[\zeta_{n}^{2} \mid \mathscr{G}_{n-1}\right]=1$ for $n \geq 0$.

Thus, $\zeta$ is a discrete-time normal noise. Note that, by letting $X=\left(X_{n}\right)$ be the partial sum sequence of $\zeta$, one gets the classical random walk.

For a discrete-time normal noise $Z=\left(Z_{n}\right)_{n \in \mathbb{N}}$ on $(\Omega, \mathscr{F}, P)$, one can construct a corresponding family $\left\{Z_{\sigma} \mid\right.$ $\sigma \in \Gamma\}$ of random variables on $(\Omega, \mathscr{F}, P)$ in the following manner:

$$
\begin{aligned}
& Z_{\emptyset}=1, \\
& Z_{\sigma}=\prod_{i \in \sigma} Z_{i},
\end{aligned}
$$

$$
\sigma \in \Gamma, \sigma \neq \emptyset \text {. }
$$

We call $\left\{Z_{\sigma} \mid \sigma \in \Gamma\right\}$ the canonical functional system of $Z$.
Lemma 2 (see $[1,2,7]$ ). Let $Z=\left(Z_{n}\right)_{n \in \mathbb{N}}$ be a discretetime normal noise on $(\Omega, \mathscr{F}, P)$. Then, its canonical functional system $\left\{Z_{\sigma} \mid \sigma \in \Gamma\right\}$ forms a countable orthonormal system in $\mathscr{L}^{2}(\Omega, \mathscr{F}, P)$.

Let $\mathscr{F}_{\infty}=\sigma\left(Z_{n} ; n \in \mathbb{N}\right)$ be the $\sigma$-field over $\Omega$ generated by a discrete-time normal noise $Z=\left(Z_{n}\right)_{n \in \mathbb{N}}$ on $(\Omega, \mathscr{F}, P)$. Then, the canonical functional system $\left\{Z_{\sigma} \mid \sigma \in \Gamma\right\}$ is also a countable orthonormal system in the space $\mathscr{L}^{2}\left(\Omega, \mathscr{F}_{\infty}, P\right)$ of square integrable complex-valued measurable functions on $\left(\Omega, \mathscr{F}_{\infty}, P\right)$.

In the literature, $\mathscr{F}_{\infty}$-measurable functions on $\Omega$ are also known as functionals of $Z$. Thus, elements of $\mathscr{L}^{2}\left(\Omega, \mathscr{F}_{\infty}, P\right)$ are naturally called square integrable functionals of $Z$.

Definition 3. A discrete-time normal noise $Z=\left(Z_{n}\right)_{n \in \mathbb{N}}$ on $(\Omega, \mathscr{F}, P)$ is said to have the chaotic representation property if its canonical functional system $\left\{Z_{\sigma} \mid \sigma \in \Gamma\right\}$ is total in $\mathscr{L}^{2}\left(\Omega, \mathscr{F}_{\infty}, P\right)$, where $\mathscr{F}_{\infty}=\sigma\left(Z_{n} ; n \in \mathbb{N}\right)$.

Thus, if a discrete-time normal noise $Z$ has the chaotic representation property, then its canonical functional system $\left\{Z_{\sigma} \mid \sigma \in \Gamma\right\}$ is actually an orthonormal basis of $\mathscr{L}^{2}\left(\Omega, \mathscr{F}_{\infty}\right.$, $P)$.

2.2. Generalized Functionals. From now on, we always assume that $Z=\left(Z_{n}\right)_{n \in \mathbb{N}}$ is a given discrete-time normal noise on $(\Omega, \mathscr{F}, P)$ that has the chaotic representation property.

For brevity, we use $\mathscr{L}^{2}(Z)$ to denote the space of square integrable functionals of $Z$; namely,

$$
\mathscr{L}^{2}(Z)=\mathscr{L}^{2}\left(\Omega, \mathscr{F}_{\infty}, P\right),
$$

where $\mathscr{F}_{\infty}=\sigma\left(Z_{n} ; n \in \mathbb{N}\right)$. For $k \geq 0$, we denote by $\mathscr{F}_{k}$ the $\sigma$-field generated by $\left(Z_{j} ; 0 \leq j \leq k\right)$; namely,

$$
\mathscr{F}_{k}=\sigma\left(Z_{j} ; 0 \leq j \leq k\right) \text {. }
$$

We note that $\mathscr{L}^{2}(Z)$ shares the same inner product $\langle\cdot, \cdot\rangle$ and norm $\|\cdot\|$ with $\mathscr{L}^{2}(\Omega, \mathscr{F}, P)$, and moreover the canonical functional system $\left\{Z_{\sigma} \mid \sigma \in \Gamma\right\}$ of $Z$ forms a countable orthonormal basis for $\mathscr{L}^{2}(Z)$, which we call the canonical orthonormal basis of $\mathscr{L}^{2}(Z)$.

Lemma 4 (see [4]). Let $\sigma \mapsto \lambda_{\sigma}$ be the $\mathbb{N}$-valued function on $\Gamma$ given by

$$
\lambda_{\sigma}= \begin{cases}\prod_{k \in \sigma}(k+1), & \sigma \neq \emptyset, \sigma \in \Gamma \\ 1, & \sigma=\emptyset, \sigma \in \Gamma .\end{cases}
$$

Then, for $p>1$, the positive term series $\sum_{\sigma \in \Gamma} \lambda_{\sigma}^{-p}$ converges and moreover

$$
\sum_{\sigma \in \Gamma} \lambda_{\sigma}^{-p} \leq \exp \left[\sum_{k=1}^{\infty} k^{-p}\right]<\infty .
$$


Using the $\mathbb{N}$-valued function defined by (8), we can construct a chain of Hilbert spaces consisting of functionals of $Z$ as follows. For $p \geq 0$, we put

$$
\mathcal{S}_{p}(Z)=\left\{\left.\xi \in \mathscr{L}^{2}(Z)\left|\sum_{\sigma \in \Gamma} \lambda_{\sigma}^{2 p}\right|\left\langle Z_{\sigma}, \xi\right\rangle\right|^{2}<\infty\right\}
$$

and define

$$
\langle\xi, \eta\rangle_{p}=\sum_{\sigma \in \Gamma} \lambda_{\sigma}^{2 p} \overline{\left\langle Z_{\sigma}, \xi\right\rangle}\left\langle Z_{\sigma}, \eta\right\rangle, \quad \xi, \eta \in \mathcal{S}_{p}(Z) .
$$

It is not hard to check that, with $\langle\cdot, \cdot\rangle_{p}$ as the inner product, $\mathcal{S}_{p}(Z)$ becomes a Hilbert space. We write $\|\xi\|_{p}=\sqrt{\langle\xi, \xi\rangle_{p}}$ for $\xi \in \mathcal{S}_{p}(Z)$. Clearly, it holds that

$$
\|\xi\|_{p}^{2}=\sum_{\sigma \in \Gamma} \lambda_{\sigma}^{2 p}\left|\left\langle Z_{\sigma}, \xi\right\rangle\right|^{2}, \quad \xi \in \mathcal{S}_{p}(Z) .
$$

Lemma 5 (see $[4,6]$ ). For $p \geq 0$, one has $\left\{Z_{\sigma} \mid \sigma \in \Gamma\right\} \subset$ $\mathcal{S}_{p}(Z)$ and moreover the system $\left\{\lambda_{\sigma}^{-p} Z_{\sigma} \mid \sigma \in \Gamma\right\}$ forms an orthonormal basis for $\mathcal{S}_{p}(Z)$.

It is easy to see that $\lambda_{\sigma} \geq 1$ for all $\sigma \in \Gamma$. This implies that $\|\cdot\|_{p} \leq\|\cdot\|_{q}$ and $\mathcal{S}_{q}(Z) \subset \mathcal{S}_{p}(Z)$ whenever $0 \leq p \leq q$. Thus, we actually get a chain of Hilbert spaces of functionals of $Z$ :

$$
\begin{aligned}
\cdots & \subset \mathcal{S}_{p+1}(Z) \subset \mathcal{S}_{p}(Z) \subset \cdots \subset \mathcal{S}_{1}(Z) \subset \mathcal{S}_{0}(Z) \\
& =\mathscr{L}^{2}(Z) .
\end{aligned}
$$

We now put

$$
\mathcal{S}(Z)=\bigcap_{p=0}^{\infty} \mathcal{S}_{p}(Z)
$$

and endow it with the topology generated by the norm sequence $\left\{\|\cdot\|_{p}\right\}_{p \geq 0}$. Note that, for each $p \geq 0, \mathcal{S}_{p}(Z)$ is just the completion of $\mathcal{S}(Z)$ with respect to $\|\cdot\|_{p}$. Thus, $\mathcal{S}(Z)$ is a countably Hilbert space $[5,8]$. The next lemma, however, shows that $\delta(Z)$ even has a much better property.

Lemma 6 (see $[4,6]$ ). The space $\mathcal{S}(Z)$ is a nuclear space; namely, for any $p \geq 0$, there exists $q>p$ such that the inclusion mapping $i_{p q}: \mathcal{S}_{q}(Z) \rightarrow \mathcal{S}_{p}(Z)$ defined by $i_{p q}(\xi)=\xi$ is a Hilbert-Schmidt operator.

For $p \geq 0$, we denote by $\mathcal{S}_{p}^{*}(Z)$ the dual of $\mathcal{S}_{p}(Z)$ and $\|\cdot\|_{-p}$ the norm of $\mathcal{S}_{p}^{*}(Z)$. Then, $\mathcal{S}_{p}^{*}(Z) \subset \mathcal{S}_{q}^{*}(Z)$ and $\|\cdot\|_{-p} \geq\|\cdot\|_{-q}$ whenever $0 \leq p \leq q$. The lemma below is then an immediate consequence of the general theory of countably Hilbert spaces (see, e.g., [8] or [5]).

Lemma 7 (see $[4,6]$ ). Let $\mathcal{S}^{*}(Z)$ be the dual of $\mathcal{S}(Z)$ and endow it with the strong topology. Then,

$$
\mathcal{S}^{*}(Z)=\bigcup_{p=0}^{\infty} \mathcal{S}_{p}^{*}(Z)
$$

and moreover the inductive limit topology over $\mathcal{S}^{*}(Z)$ given by space sequence $\left\{\mathcal{S}_{p}^{*}(Z)\right\}_{p \geq 0}$ coincides with the strong topology.
We mention that, by identifying $\mathscr{L}^{2}(Z)$ with its dual, one comes to a Gel'fand triple

$$
\mathcal{S}(Z) \subset \mathscr{L}^{2}(Z) \subset \mathcal{S}^{*}(Z),
$$

which we refer to as the Gel'fand triple associated with the discrete-time normal noise $Z$.

Theorem 8 (see [6]). The system $\left\{Z_{\sigma} \mid \sigma \in \Gamma\right\}$ is contained in $\mathcal{S}(Z)$ and moreover it forms a basis for $\mathcal{S}(Z)$ in the sense that

$$
\xi=\sum_{\sigma \in \Gamma}\left\langle Z_{\sigma}, \xi\right\rangle Z_{\sigma}, \quad \xi \in \mathcal{S}(Z)
$$

where $\langle\cdot, \cdot\rangle$ is the inner product of $\mathscr{L}^{2}(Z)$ and the series converges in the topology of $\mathcal{S}(Z)$.

Definition 9 (see $[4,6])$. Elements of $\mathcal{S}^{*}(Z)$ are called generalized functionals of $Z$, while elements of $\delta(Z)$ are called testing functionals of $Z$.

Thus, $\mathcal{S}^{*}(Z)$ and $\mathcal{S}(Z)$ can be accordingly called the generalized functional space and the testing functional space of $Z$, respectively. It turns out [6] that $\mathcal{S}^{*}(Z)$ can accommodate many quantities of theoretical interest that cannot be covered by $\mathscr{L}^{2}(Z)$.

In the following, we denote by $\langle\cdot, \cdot \cdot\rangle$ the canonical bilinear form on $\mathcal{S}^{*}(Z) \times \mathcal{S}(Z)$ given by

$$
《 \Phi, \xi 》=\Phi(\xi), \quad \Phi \in \mathcal{S}^{*}(Z), \xi \in \mathcal{S}(Z) .
$$

Note that $\langle\langle\cdot, \cdot\rangle\rangle$ is different from the inner product $\langle\cdot, \cdot\rangle$ of $\mathscr{L}^{2}(Z)$.

Definition 10 (see [6]). For $\Phi \in \mathcal{S}^{*}(Z)$, its Fock transform is the function $\widehat{\Phi}$ on $\Gamma$ given by

$$
\widehat{\Phi}(\sigma)=\left\langle\left\langle\Phi, Z_{\sigma}\right\rangle, \quad \sigma \in \Gamma,\right.
$$

where $\langle\langle\cdot, \cdot\rangle$ is the canonical bilinear form.

It is easy to verify that, for $\Phi, \Psi \in \delta^{*}(Z), \Phi=\Psi$ if and only if $\widehat{\Phi}=\widehat{\Psi}$. Thus, a generalized functional of $Z$ is completely determined by its Fock transform. The following theorem characterizes generalized functionals of $Z$ through their Fock transforms.

Theorem 11 (see [6]). Let $F$ be a function on $\Gamma$. Then, $F$ is the Fock transform of an element $\Phi$ of $\mathcal{S}^{*}(Z)$ if and only if it satisfies

$$
|F(\sigma)| \leq C \lambda_{\sigma}^{p}, \quad \sigma \in \Gamma
$$

for some constants $C \geq 0$ and $p \geq 0$. In that case, for $q>$ $p+1 / 2$, one has

$$
\|\Phi\|_{-q} \leq C\left[\sum_{\sigma \in \Gamma} \lambda_{\sigma}^{-2(q-p)}\right]^{1 / 2}
$$

and in particular $\Phi \in \mathcal{S}_{q}^{*}(Z)$.

The theorem below describes the regularity of generalized functionals of $Z$ via their Fock transforms. 
Theorem 12. Let $\Phi \in \mathcal{S}^{*}(Z)$ and $p \geq 0$. Then, $\Phi \in \mathcal{S}_{p}^{*}(Z)$ if and only if

$$
\sum_{\sigma \in \Gamma} \lambda_{\sigma}^{-2 p}|\widehat{\Phi}(\sigma)|^{2}<\infty
$$

In that case, the norm $\|\Phi\|_{-p}$ of $\Phi$ in $\mathcal{S}_{p}^{*}(Z)$ satisfies

$$
\|\Phi\|_{-p}^{2}=\sum_{\sigma \in \Gamma} \lambda_{\sigma}^{-2 p}|\widehat{\Phi}(\sigma)|^{2} .
$$

Proof. The "Only If" Part. By the well-known Riesz representation theorem [9], there exists a unique $\eta \in \mathcal{S}_{p}(Z)$ such that $\|\eta\|_{p}=\|\Phi\|_{-p}$ and

$$
\Phi(\xi)=\langle\eta, \xi\rangle_{p}, \quad \xi \in \mathcal{S}_{p}(Z) .
$$

Thus,

$$
\begin{aligned}
\sum_{\sigma \in \Gamma} \lambda_{\sigma}^{-2 p}|\widehat{\Phi}(\sigma)|^{2} & =\sum_{\sigma \in \Gamma} \lambda_{\sigma}^{-2 p}\left|\left\langle Z_{\sigma}, \eta\right\rangle_{p}\right|^{2} \\
& =\sum_{\sigma \in \Gamma} \lambda_{\sigma}^{2 p}\left|\left\langle Z_{\sigma}, \eta\right\rangle\right|^{2}=\|\eta\|_{p}^{2}=\|\Phi\|_{-p}^{2}
\end{aligned}
$$

which implies (22) and (23). have

The "If" Part. For each $\xi \in \delta(Z)$, using Theorem 8, we

$$
\begin{aligned}
& |\Phi(\xi)|=\left|\sum_{\sigma \in \Gamma}\left\langle Z_{\sigma}, \xi\right\rangle \Phi\left(Z_{\sigma}\right)\right|=\left|\sum_{\sigma \in \Gamma}\left\langle Z_{\sigma}, \xi\right\rangle \widehat{\Phi}(\sigma)\right| \\
& \quad \leq\left[\sum_{\sigma \in \Gamma} \lambda_{\sigma}^{2 p}\left|\left\langle Z_{\sigma}, \xi\right\rangle\right|^{2}\right]^{1 / 2}\left[\sum_{\sigma \in \Gamma} \lambda_{\sigma}^{-2 p}|\widehat{\Phi}(\sigma)|^{2}\right]^{1 / 2} \\
& \quad=\|\xi\|_{p}\left[\sum_{\sigma \in \Gamma} \lambda_{\sigma}^{-2 p}|\widehat{\Phi}(\sigma)|^{2}\right]^{1 / 2} .
\end{aligned}
$$

Thus, $\Phi$ is a bounded functional on the space $\left(\mathcal{S}(Z),\|\cdot\|_{p}\right)$, which implies $\Phi \in \mathcal{S}_{p}^{*}(Z)$ since $\mathcal{S}(Z)$ is dense in $\mathcal{S}_{p}(Z)$.

Remark 13. There exists a continuous linear mapping $\mathrm{R}$ : $\mathscr{L}^{2}(Z) \rightarrow \mathcal{S}^{*}(Z)$ such that

$$
\ll \mathrm{R} \eta, \xi\rangle=\langle\eta, \xi\rangle, \quad \eta \in \mathscr{L}^{2}(Z), \xi \in \mathcal{S}(Z),
$$

where $\left\langle\langle\cdot, \cdot\rangle\right.$ is the canonical bilinear form on $\mathcal{S}^{*}(Z) \times \mathcal{S}(Z)$. We call $R$ the Riesz mapping.

Theorem 14 (see [10]). Let $\Phi, \Phi_{n} \in \mathcal{S}^{*}(Z), n \geq 1$, be generalized functionals of $Z$. Then, the sequence $\left(\Phi_{n}\right)$ converges strongly to $\Phi$ in $\mathcal{S}^{*}(Z)$ if and only if it satisfies the following:

(1) $\widehat{\Phi_{n}}(\sigma) \rightarrow \widehat{\Phi}(\sigma)$ for all $\sigma \in \Gamma$.

(2) There are constants $C \geq 0$ and $p \geq 0$ such that

$$
\sup _{n \geq 1}\left|\widehat{\Phi_{n}}(\sigma)\right| \leq C \lambda_{\sigma}^{p}, \quad \sigma \in \Gamma \text {. }
$$

\section{Clark-Ocone Formula for Generalized Functionals}

In this section, we first introduce some fundamental operators on the space $\delta^{*}(Z)$. And then we establish our ClarkOcone formula for functionals in $\mathcal{S}^{*}(Z)$.

\subsection{Annihilation and Creation Operators}

Theorem 15. Let $k \in \mathbb{N}$. Then, there exists a continuous linear operator $\mathfrak{a}_{k}: \mathcal{S}^{*}(Z) \rightarrow \mathcal{S}^{*}(Z)$ such that

$$
\begin{aligned}
\widehat{\mathfrak{a}_{k} \Phi}(\sigma)=\left[1-\mathbf{1}_{\sigma}(k)\right] \widehat{\Phi}(\sigma \cup k), & \\
& \sigma \in \Gamma, \Phi \in \mathcal{S}^{*}(Z) .
\end{aligned}
$$

Proof. For each $\Phi \in \mathcal{S}^{*}(Z)$, by Theorem 11, there exist constants $C, p \geq 0$ such that

$$
|\widehat{\Phi}(\sigma)| \leq C \lambda_{\sigma}^{p}, \quad \sigma \in \Gamma
$$

which means that the function $\sigma \mapsto\left[1-\mathbf{1}_{\sigma}(k)\right] \widehat{\Phi}(\sigma \cup k)$ satisfies

$$
\begin{aligned}
& \left|\left[1-\mathbf{1}_{\sigma}(k)\right] \widehat{\Phi}(\sigma \cup k)\right| \leq\left[1-\mathbf{1}_{\sigma}(k)\right] C \lambda_{\sigma \cup k}^{p} \\
& \quad=\left[1-\mathbf{1}_{\sigma}(k)\right] C(1+k)^{p} \lambda_{\sigma}^{p} \leq C(1+k)^{p} \lambda_{\sigma}^{p} \\
& \quad \sigma \in \Gamma,
\end{aligned}
$$

which, together with Theorem 11, implies that there exists a unique $\Psi_{\Phi} \in \mathcal{S}^{*}(Z)$ such that

$$
\widehat{\Psi_{\Phi}}(\sigma)=\left[1-\mathbf{1}_{\sigma}(k)\right] \widehat{\Phi}(\sigma \cup k), \quad \sigma \in \Gamma .
$$

Now, consider the mapping $\mathfrak{a}_{k}: \mathcal{S}^{*}(Z) \rightarrow \mathcal{S}^{*}(Z)$ defined by

$$
\mathfrak{a}_{k} \Phi=\Psi_{\Phi}, \quad \Phi \in \mathcal{S}^{*}(Z) .
$$

It is not hard to verify that $\mathfrak{a}_{k}$ is a linear operator and satisfies (29). To complete the proof, we still need to show that $\mathfrak{a}_{k}$ : $\mathcal{S}^{*}(Z) \rightarrow \mathcal{S}^{*}(Z)$ is continuous with respect to the strong topology over $\mathcal{S}^{*}(Z)$.

Let $p \geq 0$ and denote by $\dot{\mathrm{i}}_{k}: \mathcal{S}_{p}^{*}(Z) \rightarrow \mathcal{S}^{*}(Z)$ the inclusion mapping; namely, $\dot{\mathfrak{j}}_{k}$ is the mapping defined by

$$
\dot{\mathrm{j}}_{k}(\Phi)=\Phi, \quad \Phi \in \mathcal{S}_{p}^{*}(Z)
$$

Then, the composition mapping $\mathfrak{a}_{k} \circ \dot{\mathfrak{j}}_{k}$ is a linear operator from $\mathcal{S}_{p}^{*}(Z)$ to $\mathcal{S}^{*}(Z)$. For each $\Phi \in \mathcal{S}_{p}^{*}(Z)$, we have

$$
\begin{gathered}
\sum_{\sigma \in \Gamma} \lambda_{\sigma}^{-2 p}\left|\mathfrak{a}_{k} \widehat{\circ \dot{\mathfrak{j}}_{k}(\Phi)}(\sigma)\right|^{2}=\sum_{\sigma \in \Gamma} \lambda_{\sigma}^{-2 p}\left|\widehat{\mathfrak{a}_{k} \Phi}(\sigma)\right|^{2} \\
=\sum_{\sigma \in \Gamma} \lambda_{\sigma}^{-2 p}\left|\left[1-\mathbf{1}_{\sigma}(k)\right] \widehat{\Phi}(\sigma \cup k)\right|^{2} \\
=\sum_{k \notin \sigma \in \Gamma}(1+k)^{2 p} \lambda_{\sigma \cup k}^{-2 p}|\widehat{\Phi}(\sigma \cup k)|^{2} \\
\leq(1+k)^{2 p} \sum_{\tau \in \Gamma} \lambda_{\tau}^{-2 p}|\widehat{\Phi}(\tau)|^{2}
\end{gathered}
$$


which together with Theorem 12 implies that $\mathfrak{a}_{k} \circ \dot{\mathfrak{j}}_{k}(\Phi) \epsilon$ $\mathcal{S}_{p}^{*}(Z)$ and

$$
\left\|\mathfrak{a}_{k} \circ \dot{\mathfrak{j}}_{k}(\Phi)\right\|_{-p} \leq(1+k)^{p}\|\Phi\|_{-p} .
$$

Thus, $\mathfrak{a}_{k} \circ \dot{\mathfrak{j}}_{k}\left(\mathcal{S}_{p}^{*}(Z)\right) \subset \mathcal{S}_{p}^{*}(Z)$ and $\mathfrak{a}_{k} \circ \dot{\mathfrak{j}}_{k}: \mathcal{S}_{p}^{*}(Z) \rightarrow \mathcal{S}_{p}^{*}(Z)$ is a bounded operator, which implies that $\mathfrak{a}_{k} \circ \dot{\mathfrak{j}}_{k}$ is continuous as an operator from $\mathcal{S}_{p}^{*}(Z)$ to $\mathcal{S}^{*}(Z)$.

Since the choice of the above $p \geq 0$ is arbitrary, we actually arrive at a conclusion that the composition mapping $\mathfrak{a}_{k} \circ \dot{\mathfrak{j}}_{k}: \mathcal{S}_{p}^{*}(Z) \rightarrow \mathcal{S}^{*}(Z)$ is continuous for all $p \geq 0$. Therefore, $\mathfrak{a}_{k}: \mathcal{S}^{*}(Z) \rightarrow \mathcal{S}^{*}(Z)$ is continuous with respect to the inductive limit topology over $\mathcal{S}^{*}(Z)$, which together with Lemma 7 implies that $\mathfrak{a}_{k}: \mathcal{S}^{*}(Z) \rightarrow \mathcal{S}^{*}(Z)$ is continuous with respect to the strong topology over $\mathcal{S}^{*}(Z)$.

Carefully checking the proof of Theorem 15 , one can find the next result already proven.

Theorem 16. Let $k \in \mathbb{N}$. Then, for each $p \geq 0, \mathcal{S}_{p}^{*}(Z)$ keeps invariant under the action of $\mathfrak{a}_{k}$, and moreover

$$
\left\|\mathfrak{a}_{k} \Phi\right\|_{-p} \leq(1+k)^{p}\|\Phi\|_{-p}, \quad \Phi \in \mathcal{S}_{p}^{*}(Z) .
$$

With the same arguments, we can prove the next two theorems, which are dual forms of Theorems 15 and 16, respectively.

Theorem 17. Let $k \in \mathbb{N}$. Then, there exists a continuous linear operator $\mathfrak{a}_{k}^{\dagger}: \mathcal{S}^{*}(Z) \rightarrow \mathcal{S}^{*}(Z)$ such that

$$
\widehat{\mathfrak{a}_{k}^{\dagger} \Phi}(\sigma)=\mathbf{1}_{\sigma}(k) \widehat{\Phi}(\sigma \backslash k), \quad \sigma \in \Gamma, \Phi \in \mathcal{S}^{*}(Z) .
$$

Proof. For each $\Phi \in \mathcal{S}^{*}(Z)$, by Theorem 11, there exist constants $C, p \geq 0$ such that

$$
|\widehat{\Phi}(\sigma)| \leq C \lambda_{\sigma}^{p}, \quad \sigma \in \Gamma,
$$

which means that the function $\sigma \mapsto \mathbf{1}_{\sigma}(k) \widehat{\Phi}(\sigma \backslash k)$ satisfies

$$
\begin{aligned}
\left|\mathbf{1}_{\sigma}(k) \widehat{\Phi}(\sigma \backslash k)\right| & \leq \mathbf{1}_{\sigma}(k) C \lambda_{\sigma \backslash k}^{p} \\
& =\mathbf{1}_{\sigma}(k) C(1+k)^{-p} \lambda_{\sigma}^{p} \\
& \leq C(1+k)^{-p} \lambda_{\sigma}^{p}, \quad \sigma \in \Gamma,
\end{aligned}
$$

which, together with Theorem 11, implies that there exists a unique $\Theta_{\Phi} \in \mathcal{S}^{*}(Z)$ such that

$$
\widehat{\Theta_{\Phi}}(\sigma)=\mathbf{1}_{\sigma}(k) \widehat{\Phi}(\sigma \backslash k), \quad \sigma \in \Gamma .
$$

Now, consider the mapping $\mathfrak{a}_{k}^{\dagger}: \mathcal{S}^{*}(Z) \rightarrow \mathcal{S}^{*}(Z)$ defined by

$$
\mathfrak{a}_{k}^{\dagger} \Phi=\Theta_{\Phi}, \quad \Phi \in \mathcal{S}^{*}(Z) .
$$

It is not hard to verify that $\mathfrak{a}_{k}^{\dagger}$ is a linear operator and satisfies (38). To complete the proof, we still need to show that $\mathfrak{a}_{k}^{\dagger}$ : $\mathcal{S}^{*}(Z) \rightarrow \mathcal{S}^{*}(Z)$ is continuous with respect to the strong topology over $\mathcal{S}^{*}(Z)$.
Let $p \geq 0$ and denote by $\dot{\mathfrak{j}}_{k}: \mathcal{S}_{p}^{*}(Z) \rightarrow \mathcal{S}^{*}(Z)$ the inclusion mapping. Then, the composition mapping $\mathfrak{a}_{k}^{\dagger} \circ \dot{\mathfrak{j}}_{k}$ is a linear operator from $\mathcal{S}_{p}^{*}(Z)$ to $\mathcal{S}^{*}(Z)$. For each $\Phi \in \mathcal{S}_{p}^{*}(Z)$, we have

$$
\begin{aligned}
& \sum_{\sigma \in \Gamma} \lambda_{\sigma}^{-2 p}\left|\widehat{\mathfrak{a}_{k}^{\dagger} \widehat{\dot{\mathbf{j}}_{k}}(\Phi)}(\sigma)\right|^{2}=\sum_{\sigma \in \Gamma} \lambda_{\sigma}^{-2 p}\left|\widehat{\mathfrak{a}_{k}^{\dagger} \Phi}(\sigma)\right|^{2} \\
& \quad=\sum_{\sigma \in \Gamma} \lambda_{\sigma}^{-2 p}\left|\mathbf{1}_{\sigma}(k) \widehat{\Phi}(\sigma \backslash k)\right|^{2} \\
& \quad=\sum_{k \in \sigma \in \Gamma}(1+k)^{-2 p} \lambda_{\sigma \backslash k}^{-2 p}|\widehat{\Phi}(\sigma \backslash k)|^{2} \\
& \quad \leq(1+k)^{-2 p} \sum_{\tau \in \Gamma} \lambda_{\tau}^{-2 p}|\widehat{\Phi}(\tau)|^{2},
\end{aligned}
$$

which together with Theorem 12 implies that $\mathfrak{a}_{k}^{\dagger} \circ \dot{\mathfrak{j}}_{k}(\Phi) \epsilon$ $\mathcal{S}_{p}^{*}(Z)$ and

$$
\left\|\mathfrak{a}_{k}^{\dagger} \circ \dot{\mathfrak{j}}_{k}(\Phi)\right\|_{-p} \leq(1+k)^{-p}\|\Phi\|_{-p}
$$

Thus, $\mathfrak{a}_{k}^{\dagger} \circ \dot{\dot{j}}_{k}\left(\mathcal{S}_{p}^{*}(Z)\right) \subset \mathcal{S}_{p}^{*}(Z)$ and $\mathfrak{a}_{k}^{\dagger} \circ \dot{\mathfrak{j}}_{k}: \mathcal{S}_{p}^{*}(Z) \rightarrow \mathcal{S}_{p}^{*}(Z)$ is a bounded operator, which implies that $\mathfrak{a}_{k}^{\dagger} \circ \dot{\mathbf{j}}_{k}$ is continuous as an operator from $\mathcal{S}_{p}^{*}(Z)$ to $\mathcal{S}^{*}(Z)$.

Since the choice of the above $p \geq 0$ is arbitrary, we actually arrive at a conclusion that the composition mapping $\mathfrak{a}_{k}^{\dagger} \circ \dot{\mathfrak{j}}_{k}: \mathcal{S}_{p}^{*}(Z) \rightarrow \mathcal{S}^{*}(Z)$ is continuous for all $p \geq 0$. Therefore, $\mathfrak{a}_{k}^{\dagger}: \mathcal{S}^{*}(Z) \rightarrow \mathcal{S}^{*}(Z)$ is continuous with respect to the inductive limit topology over $\delta^{*}(Z)$, which together with Lemma 7 implies that $\mathfrak{a}_{k}^{\dagger}: \mathcal{S}^{*}(Z) \rightarrow \mathcal{S}^{*}(Z)$ is continuous with respect to the strong topology over $\mathcal{S}^{*}(Z)$.

From the proof of Theorem 17, we can easily get the next result concerning the operator $\mathfrak{a}_{k}^{\dagger}$.

Theorem 18. Let $k \in \mathbb{N}$. Then, for each $p \geq 0, \mathcal{S}_{p}^{*}(Z)$ keeps invariant under the action of $\mathfrak{a}_{k}^{\dagger}$, and moreover

$$
\left\|\mathfrak{a}_{k}^{\dagger} \Phi\right\|_{-p} \leq(1+k)^{-p}\|\Phi\|_{-p}, \quad \Phi \in \mathcal{S}_{p}^{*}(Z) .
$$

Remark 19. For $k \geq 0$, the corresponding annihilation operator $\partial_{k}$ on $\mathscr{L}^{2}(Z)$ and its dual $\partial_{k}^{*}$ (known as the creation operator) admit the property

$$
\begin{aligned}
& \partial_{k} Z_{\sigma}=\mathbf{1}_{\sigma}(k) Z_{\sigma \backslash k}, \\
& \partial_{k}^{*} Z_{\sigma}=\left[1-\mathbf{1}_{\sigma}(k)\right] Z_{\sigma \cup k},
\end{aligned}
$$

$$
\sigma \in \Gamma
$$

And moreover, they satisfy the canonical anticommutation relation (CAR) in equal-time

$$
\partial_{k}^{*} \partial_{k}+\partial_{k} \partial_{k}^{*}=I,
$$

where $I$ means the identity operator on $\mathscr{L}^{2}(Z)$. We refer to $[2,6]$ and for details about these operators.

The next theorem shows the link between $\mathfrak{a}_{k}$ and $\partial_{k}$, as well as between $\mathfrak{a}_{k}^{\dagger}$ and $\partial_{k}^{*}$. 
Theorem 20. Let $k \geq 0$. Then, the operators $\mathfrak{a}_{k}$ and $\mathfrak{a}_{k}^{\dagger}$ satisfy

$$
\begin{aligned}
& \mathfrak{a}_{k} \mathrm{R}=\mathrm{R} \partial_{k}, \\
& \mathfrak{a}_{k}^{\dagger} \mathrm{R}=\mathrm{R} \partial_{k}^{*},
\end{aligned}
$$

where $\mathrm{R}$ is the Riesz mapping as indicated in Remark 13.

Proof. Let $\eta \in \mathscr{L}^{2}(Z)$. Then, for all $\sigma \in \Gamma$, we have

$$
\begin{aligned}
\widehat{\mathfrak{a}_{k} \mathrm{R} \eta}(\sigma) & =\left[1-\mathbf{1}_{\sigma}(k)\right]\left\langle\eta, Z_{\sigma \cup k}\right\rangle=\left\langle\eta, \partial_{k}^{*} Z_{\sigma}\right\rangle \\
& =\left\langle\partial_{k} \eta, Z_{\sigma}\right\rangle=\widehat{\operatorname{R} \partial_{k} \eta}(\sigma),
\end{aligned}
$$

which implies $\mathfrak{a}_{k} \mathrm{R} \eta=\mathrm{R} \partial_{k} \eta$. It then follows by the arbitrariness of $\eta \in \mathscr{L}^{2}(Z)$ that $\mathfrak{a}_{k} \mathrm{R}=\mathrm{R} \partial_{k}$. Similarly, we can prove $\mathfrak{a}_{k}^{\dagger} \mathrm{R}=\mathrm{R} \partial_{k}^{*}$.

In view of Theorem 20, we give the following definition to name the operators $\mathfrak{a}_{k}$ and $\mathfrak{a}_{k}^{\dagger}$.

Definition 21. For $k \geq 0$, the operators $\mathfrak{a}_{k}$ and $\mathfrak{a}_{k}^{\dagger}$ are called the annihilation and creation operators on generalized functionals of $Z$, respectively.

Much like the operators $\left\{\partial_{k}, \partial_{k}^{*}\right\}$ on $\mathscr{L}^{2}(Z)$, the operators $\left\{\mathfrak{a}_{k}, \mathfrak{a}_{k}^{\dagger}\right\}$ also satisfy a canonical anticommutation relation (CAR) in equal-time.

Theorem 22. Let I be the identity operator on $\mathcal{S}^{*}(Z)$. Then, for $k \geq 0$, it holds that

$$
\mathfrak{a}_{k}^{\dagger} \mathfrak{a}_{k}+\mathfrak{a}_{k} \mathfrak{a}_{k}^{\dagger}=I
$$

Proof. Let $\Phi \in \mathcal{S}^{*}(Z)$. Then, for any $\sigma \in \Gamma$, it follows from (29) and (38) that

$$
\begin{aligned}
\widehat{\mathfrak{a}_{k}^{\dagger} \mathfrak{a}_{k} \Phi}(\sigma) & =\mathbf{1}_{\sigma}(k) \widehat{\mathfrak{a}_{k} \Phi}(\sigma \backslash k)=\mathbf{1}_{\sigma}(k) \widehat{\Phi}(\sigma), \\
\widehat{\mathfrak{a}_{k} \mathfrak{a}_{k}^{\dagger} \Phi}(\sigma) & =\left(1-\mathbf{1}_{\sigma}(k)\right) \widehat{\mathfrak{a}_{k}^{\dagger} \Phi}(\sigma \cup k) \\
& =\left(1-\mathbf{1}_{\sigma}(k)\right) \widehat{\Phi}(\sigma),
\end{aligned}
$$

and thus

$$
\begin{aligned}
\left(\mathfrak{a}_{k}^{\dagger} \widehat{\mathfrak{a}_{k}+\mathfrak{a}_{k}} \mathfrak{a}_{k}^{\dagger}\right) \Phi(\sigma) & =\widehat{\mathfrak{a}_{k} \mathfrak{a}_{k}^{\dagger} \Phi}(\sigma)+\widehat{\mathfrak{a}_{k} \mathfrak{a}_{k}^{\dagger} \Phi}(\sigma) \\
& =\widehat{\Phi}(\sigma)
\end{aligned}
$$

which implies that $\left(\mathfrak{a}_{k}^{\dagger} \mathfrak{a}_{k}+\mathfrak{a}_{k} \mathfrak{a}_{k}^{\dagger}\right) \Phi=\Phi$. It then follows from the arbitrariness of $\Phi \in \mathcal{S}^{*}(Z)$ that $\mathfrak{a}_{k}^{\dagger} \mathfrak{a}_{k}+\mathfrak{a}_{k} \mathfrak{a}_{k}^{\dagger}=I$.

3.2. Expectation and Conditional Expectation Operators. For the Riesz mapping $\mathrm{R}$, using Theorem 12, we can prove that $\mathrm{R} \eta \in \mathcal{S}_{0}^{*}(Z)$ for all $\eta \in \mathscr{L}^{2}(Z)$. In particular, we have R1 $\epsilon$ $\delta_{0}^{*}(Z)$.

Theorem 23. The mapping $\mathfrak{E}: \mathcal{S}^{*}(Z) \rightarrow \mathcal{S}^{*}(Z)$ defined by

$$
\mathfrak{F} \Phi=\widehat{\Phi}(\emptyset) \mathrm{R} 1, \quad \Phi \in \mathcal{S}^{*}(Z),
$$

is a continuous linear operator from $\mathcal{S}^{*}(Z)$ to itself. And, moreover,

$$
\widehat{\mathfrak{F} \Phi}(\sigma)=\widehat{\Phi}(\emptyset)\left\langle 1, Z_{\sigma}\right\rangle, \quad \sigma \in \Gamma, \Phi \in \mathcal{S}^{*}(Z) .
$$

Proof. Clearly, $\mathfrak{E}: \mathcal{S}^{*}(Z) \rightarrow \mathcal{S}^{*}(Z)$ is a linear operator and satisfies (54). Next, let us show that $\mathfrak{E}: \mathcal{S}^{*}(Z) \rightarrow \mathcal{S}^{*}(Z)$ is continuous with respect to the strong topology over $\mathcal{S}^{*}(Z)$.

Let $p \geq 0$ and denote by $\dot{\mathrm{i}}_{k}: \mathcal{\delta}_{p}^{*}(Z) \rightarrow \mathcal{S}^{*}(Z)$ the inclusion mapping. Then, the composition mapping $\mathfrak{F} \circ \dot{j}_{k}$ is a linear operator from $\mathcal{S}_{p}^{*}(Z)$ to $\mathcal{S}^{*}(Z)$. For each $\Phi \in \mathcal{S}_{p}^{*}(Z)$, we have

$$
\begin{gathered}
\sum_{\sigma \in \Gamma} \lambda_{\sigma}^{-2 p}\left|\widehat{\mathfrak{F} \circ \dot{\mathfrak{j}}_{k}(\Phi)}(\sigma)\right|^{2}=\sum_{\sigma \in \Gamma} \lambda_{\sigma}^{-2 p}|\widehat{\mathfrak{E} \Phi}(\sigma)|^{2} \\
\quad=\sum_{\sigma \in \Gamma} \lambda_{\sigma}^{-2 p}\left|\widehat{\Phi}(\emptyset)\left\langle 1, Z_{\sigma}\right\rangle\right|^{2} \leq \sum_{\sigma \in \Gamma} \lambda_{\sigma}^{-2 p}|\widehat{\Phi}(\sigma)|^{2}
\end{gathered}
$$

which together with Theorem 12 implies that $\mathfrak{E} \circ \dot{\mathfrak{j}}_{k}(\Phi) \epsilon$ $\mathcal{S}_{p}^{*}(Z)$ and

$$
\left\|\mathfrak{E} \circ \dot{\mathfrak{I}}_{k}(\Phi)\right\|_{-p} \leq\|\Phi\|_{-p} .
$$

Thus, $\mathfrak{F} \circ \dot{\mathfrak{l}}_{k}\left(\mathcal{S}_{p}^{*}(Z)\right) \subset \mathcal{S}_{p}^{*}(Z)$ and $\mathfrak{E} \circ \dot{\mathfrak{j}}_{k}: \mathcal{S}_{p}^{*}(Z) \rightarrow \mathcal{S}_{p}^{*}(Z)$ is a bounded operator, which implies that $\mathfrak{E} \circ \dot{\mathfrak{j}}_{k}$ is continuous as an operator from $\mathcal{S}_{p}^{*}(Z)$ to $\mathcal{S}^{*}(Z)$.

Since the choice of the above $p \geq 0$ is arbitrary, we actually arrive at a conclusion that the composition mapping $\mathfrak{E} \circ \dot{\mathrm{j}}_{k}: \mathcal{S}_{p}^{*}(Z) \rightarrow \mathcal{S}^{*}(Z)$ is continuous for all $p \geq 0$. Therefore, $\mathfrak{F}^{*}: \mathcal{S}^{*}(Z) \rightarrow \mathcal{S}^{*}(Z)$ is continuous with respect to the inductive limit topology over $\mathcal{S}^{*}(Z)$, which together with Lemma 7 implies that $\mathfrak{F}: \mathcal{S}^{*}(Z) \rightarrow \mathcal{S}^{*}(Z)$ is continuous with respect to the strong topology over $\mathcal{S}^{*}(Z)$.

Definition 24. The operator $\mathfrak{E}$ is called the expectation operator on generalized functionals of $Z$.

Since $1 \in \mathscr{L}^{2}(Z)$, the expectation $\mathbb{E}$ with respect to $P$ is actually a bounded operator from $\mathscr{L}^{2}(Z)$ to itself. The next theorem shows the link between the operators $\mathfrak{E}$ and $\mathbb{E}$, which justifies the above definition.

Theorem 25. It holds that $\mathfrak{E} \mathrm{R}=\mathrm{R} \mathbb{E}$, where $\mathrm{R}$ is the Riesz mapping.

Proof. For any $\xi \in \mathscr{L}^{2}(Z)$ and any $\sigma \in \Gamma$, by a direct computation, we have

$$
\begin{aligned}
\widehat{\operatorname{RE} \xi}(\sigma) & =\left\langle\mathbb{E} \xi, Z_{\sigma}\right\rangle=\left\langle\xi, Z_{\emptyset}\right\rangle\left\langle 1, Z_{\sigma}\right\rangle \\
& =\widehat{\operatorname{R} \xi}(\emptyset)\left\langle 1, Z_{\sigma}\right\rangle=\widehat{\mathfrak{E R} \xi}(\sigma) .
\end{aligned}
$$

Thus, $\mathfrak{E} R=\mathrm{R} \mathbb{E}$.

Theorem 26. Let $k \geq 0$. Then, there exists a continuous linear operator $\mathfrak{F}_{k}: \mathcal{S}^{*}(Z) \rightarrow \mathcal{S}^{*}(Z)$ such that

$$
\widehat{\mathfrak{E}_{k} \Phi}(\sigma)=\mathbf{1}_{\Gamma_{k]}}(\sigma) \widehat{\Phi}(\sigma), \quad \sigma \in \Gamma,
$$

where $\Gamma_{k]}=\{\sigma \in \Gamma \mid \max \sigma \leq k\}$ and $\mathbf{1}_{\Gamma_{k]}}(\cdot)$ denotes the indicator of $\Gamma_{k]}$. 
Proof. We omit the proof because it is quite similar to that of Theorem 15.

Using Theorems 12 and 26, we can easily prove the next theorem, which shows that the operator $\mathfrak{F}_{k}$ has a type of contraction property on $\mathcal{S}^{*}(Z)$.

Theorem 27. Let $k \geq 0$. Then, for each $p \geq 0$, $\mathcal{S}_{p}^{*}(Z)$ keeps invariant under the action of $\mathfrak{F}_{k}$, and moreover

$$
\left\|\mathfrak{F}_{k} \Phi\right\|_{-p} \leq\|\Phi\|_{-p}, \quad \forall \Phi \in \mathcal{S}_{p}^{*}(Z)
$$

Definition 28. The operators $\mathfrak{F}_{k}, k \geq 0$, are called the conditional expectation operators on generalized functionals of $Z$.

For $k \geq 0$, we set $P_{k}=\mathbb{E}\left[\cdot \mid \mathscr{F}_{k}\right]$, the expectation given $\mathscr{F}_{k}$, where $\mathscr{F}_{k}$ is the $\sigma$-field generated by $\left(Z_{j} ; 0 \leq j \leq k\right)$ as mentioned above. $P_{k}$ is usually known as a conditional expectation operator on square integrable functionals of $Z$. The theorem below then justifies Definition 28.

Theorem 29. For each $k \geq 0$, it holds that $\mathfrak{F}_{k} \mathrm{R}=\mathrm{R} P_{k}$, where $\mathrm{R}$ is the Riesz mapping.

Proof. Let $k \geq 0$. Then, for any $\xi \in \mathscr{L}^{2}(Z)$ and any $\sigma \in \Gamma$, by a direct computation, we have

$$
\begin{aligned}
\widehat{\operatorname{RP}_{k} \xi}(\sigma) & =\left\langle P_{k} \xi, Z_{\sigma}\right\rangle=\left\langle\xi, P_{k} Z_{\sigma}\right\rangle=\mathbf{1}_{\Gamma_{k]}}(\sigma)\left\langle\xi, Z_{\sigma}\right\rangle \\
& =\mathbf{1}_{\Gamma_{k]}}(\sigma) \widehat{\operatorname{R\xi }}(\sigma)=\widehat{\mathfrak{E}_{k} \mathrm{R} \xi}(\sigma) .
\end{aligned}
$$

Thus, $\mathfrak{F}_{k} \mathrm{R}=\mathrm{R} P_{k}$.

3.3. Clark-Ocone Formula for Generalized Functionals. In this subsection, we establish our Clark-Ocone formula for generalized functionals of $Z$.

Theorem 30. For all generalized functionals $\Phi \in \mathcal{S}^{*}(Z)$, it holds that

$$
\Phi=\mathfrak{E} \Phi+\sum_{k=0}^{\infty} \mathfrak{F}_{k} \mathfrak{a}_{k}^{\dagger} \mathfrak{a}_{k} \Phi
$$

where the series on the right-hand side converges strongly in $\mathcal{S}^{*}(Z)$.

Proof. Let $\Phi \in \mathcal{S}^{*}(Z)$ and $\Psi_{n}=\sum_{k=0}^{n} \mathfrak{\mathfrak { F }}_{k} \mathfrak{a}_{k}^{\dagger} \mathfrak{a}_{k} \Phi$ for $n \geq 0$. Then, for $\sigma \in \Gamma$, by a direct computation, we have

$$
\begin{aligned}
\widehat{\Psi_{n}}(\sigma) & =\sum_{k=0}^{n} \mathbf{1}_{\Gamma_{k]}}(\sigma) \mathbf{1}_{\sigma}(k) \widehat{\Phi}(\sigma) \\
& = \begin{cases}0, & \sigma=\emptyset ; \\
0, & \sigma \neq \emptyset, n<\max \sigma \\
\widehat{\Phi}(\sigma), & \sigma \neq \emptyset, n \geq \max \sigma .\end{cases}
\end{aligned}
$$

It then follows that $\widehat{\Psi_{n}}(\sigma) \rightarrow \widehat{\Phi-\mathfrak{E}} \Phi(\sigma)$ for all $\sigma \in \Gamma$ as $n \rightarrow$ $\infty$. On the other hand, by Theorem 11 , there are constants $C \geq$ 0 and $p \geq 0$ such that

$$
|\widehat{\Phi}(\sigma)| \leq C \lambda_{\sigma}^{p}, \quad \sigma \in \Gamma,
$$

which together with (62) gives

$$
\sup _{n \geq 0}\left|\widehat{\Psi}_{n}(\sigma)\right| \leq|\widehat{\Phi}(\sigma)| \leq C \lambda_{\sigma}^{p}, \quad \sigma \in \Gamma .
$$

Therefore, by Theorem 14, we know $\left(\Psi_{n}\right)$ converges strongly to $\Phi-\mathfrak{E} \Phi$ in $\mathcal{S}^{*}(Z)$. This completes the proof.

Proposition 31. For each $k \geq 0$, it holds that

$$
\begin{aligned}
& \mathfrak{\mathfrak { E }}_{k} \mathfrak{a}_{k}^{\dagger}=\mathfrak{a}_{k}^{\dagger} \mathfrak{\mathfrak { E }}_{k}, \\
& \mathfrak{\mathfrak { E }}_{k} \mathfrak{a}_{k}=\mathfrak{\mathfrak { E }}_{k-1} \mathfrak{a}_{k},
\end{aligned}
$$

where $\mathfrak{F}_{-1}=\mathfrak{F}$.

Proof. Let $k \geq 0$. Then, for all $\Phi \in \mathcal{S}^{*}(Z)$ and $\sigma \in \Gamma$, by Theorems 17 and 26, we get

$$
\begin{aligned}
\widehat{\mathfrak{E}_{k} \mathfrak{a}_{k}^{\dagger} \Phi}(\sigma) & =\mathbf{1}_{\Gamma_{k]}}(\sigma) \mathbf{1}_{\sigma}(k) \widehat{\Phi}(\sigma \backslash k) \\
& =\mathbf{1}_{\Gamma_{k]}}(\sigma \backslash k) \mathbf{1}_{\sigma}(k) \widehat{\Phi}(\sigma \backslash k) \\
& =\widehat{\mathfrak{a}_{k}^{\dagger} \mathfrak{\xi}_{k} \Phi}(\sigma),
\end{aligned}
$$

where equality $\mathbf{1}_{\Gamma_{k]}}(\sigma) \mathbf{1}_{\sigma}(k)=\mathbf{1}_{\Gamma_{k]}}(\sigma \backslash k) \mathbf{1}_{\sigma}(k)$ is used. Thus,

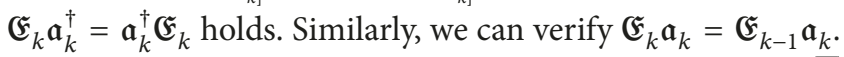

Combining Theorem 30 with Proposition 31, we arrive at the next interesting result, which we call the Clark-Ocone formula for generalized functionals of $Z$.

Theorem 32. For all generalized functionals $\Phi \in \mathcal{S}^{*}(Z)$, it holds that

$$
\Phi=\mathfrak{E} \Phi+\sum_{k=0}^{\infty} \mathfrak{a}_{k}^{\dagger} \mathfrak{\mathfrak { r }}_{k-1} \mathfrak{a}_{k} \Phi,
$$

where $\mathfrak{E}_{-1}=\mathfrak{E}$ and the series on the right-hand side converges strongly in $\mathcal{S}^{*}(Z)$.

Remark 33. As mentioned above, $\partial_{k}$ and $\partial_{k}^{*}$ are the annihilation and creation operators on $\mathscr{L}^{2}(Z)$, respectively, and $P_{k}=\mathbb{E}\left[\cdot \mid \mathscr{F}_{k}\right]$ is the conditional expectation operator on $\mathscr{L}^{2}(Z)$. It can be verified that

$$
\partial_{k}^{*} P_{k-1} \eta=Z_{k} P_{k-1} \eta, \quad \forall k \geq 0, \forall \eta \in \mathscr{L}^{2}(Z),
$$

where $P_{-1}=\mathbb{E}$ and $Z_{k}$ is the $k$-component of the discretetime normal noise $Z$. Thus, the Clark-Ocone formula (1) can be rewritten as the following form:

$$
\xi=\mathbb{E} \xi+\sum_{k=0}^{\infty} \partial_{k}^{*} P_{k-1} \partial_{k} \xi, \quad \xi \in \mathscr{L}^{2}(Z),
$$

where the series on the right-hand side converges in the norm of $\mathscr{L}^{2}(Z)$. This observation justifies calling formula (67) the Clark-Ocone formula for generalized functionals of $Z$. 


\section{Applications}

In the final section, we show some applications of our ClarkOcone formula.

For $p \geq 0$ and $\Phi, \Psi \in \mathcal{S}^{*}(Z)$, we define $\langle\Phi, \Psi\rangle_{-p}$ as

$$
\langle\Phi, \Psi\rangle_{-p}=\sum_{\sigma \in \Gamma} \lambda_{\sigma}^{-2 p} \widehat{\Phi}(\sigma) \overline{\widehat{\Psi}(\sigma)}
$$

provided the series on the right-hand side absolutely converges. Note that if $\Phi, \Psi \in \mathcal{S}_{p}^{*}(Z)$, then by Theorem 12 the series in (70) absolutely converges, and hence $\langle\Phi, \Psi\rangle_{-p}$ makes sense, and in particular

$$
\langle\Phi, \Phi\rangle_{-p}=\|\Phi\|_{-p}^{2}
$$

Definition 34. For generalized functionals $\Phi, \Psi \in \mathcal{S}^{*}(Z)$, their $p$-covariant $\operatorname{cov}_{p}(\Phi, \Psi), p \geq 0$, is defined as

$$
\operatorname{cov}_{p}(\Phi, \Psi)=\langle\Phi-\mathfrak{E} \Phi, \Psi-\mathfrak{E} \Psi\rangle_{-p}
$$

provided the right-hand side makes sense.

By convention, $\operatorname{var}_{p}(\Phi) \equiv \operatorname{cov}_{p}(\Phi, \Phi)$ is called the $p$ variant of generalized functional $\Phi$. Clearly, $\operatorname{var}_{p}(\Phi)=\| \Phi-$ $\mathfrak{E} \Phi \|_{-p}^{2}$ if $\Phi \in \mathcal{S}_{p}^{*}(Z)$.

Theorem 35. Let $\Phi, \Psi \in \mathcal{S}_{p}^{*}(Z)$ for some $p \geq 0$. Then, their p-covariant $\operatorname{cov}_{p}(\Phi, \Psi)$ makes sense, and moreover

$$
\operatorname{cov}_{p}(\Phi, \Psi)=\sum_{k=0}^{\infty}\left\langle\mathfrak{F}_{k} \mathfrak{a}_{k}^{\dagger} \mathfrak{a}_{k} \Phi, \mathfrak{F}_{k} \mathfrak{a}_{k}^{\dagger} \mathfrak{a}_{k} \Psi\right\rangle_{-p}
$$

Proof. By Theorem 12, the series on the right-hand side of (73) converges absolutely. On the other hand, by Theorem 30, we have

$$
\begin{aligned}
\operatorname{cov}_{p}(\Phi, \Psi)= & \sum_{\sigma \in \Gamma} \lambda_{\sigma}^{-2 p} \widehat{\Phi-\mathscr{E} \Phi}(\sigma) \overline{\Psi-\mathscr{\Psi} \Psi}(\sigma) \\
= & \sum_{\sigma \in \Gamma} \lambda_{\sigma}^{-2 p}\left[\sum_{k=0}^{\infty} \mathbf{1}_{\Gamma_{k]}}(\sigma) \mathbf{1}_{\sigma}(k) \widehat{\Phi}(\sigma)\right] \\
& \cdot\left[\sum_{k=0}^{\infty} \mathbf{1}_{\Gamma_{k]}}(\sigma) \mathbf{1}_{\sigma}(k) \overline{\widehat{\Psi}(\sigma)}\right]
\end{aligned}
$$

which together with the fact

$$
\mathbf{1}_{\Gamma_{j]}}(\sigma) \mathbf{1}_{\sigma}(j) \mathbf{1}_{\Gamma_{k]}}(\sigma) \mathbf{1}_{\sigma}(k)=0,
$$

$$
j \neq k, j, k \geq 0, \sigma \in \Gamma
$$

gives

$$
\begin{aligned}
& \operatorname{cov}_{p}(\Phi, \Psi)=\sum_{\sigma \in \Gamma} \lambda_{\sigma}^{-2 p} \sum_{k=0}^{\infty} \mathbf{1}_{\Gamma_{k]}}(\sigma) \mathbf{1}_{\sigma}(k) \widehat{\Phi}(\sigma) \overline{\widehat{\Psi}(\sigma)} \\
& =\sum_{k=0}^{\infty} \sum_{\sigma \in \Gamma} \lambda_{\sigma}^{-2 p}\left[\mathbf{1}_{\Gamma_{k]}}(\sigma) \mathbf{1}_{\sigma}(k) \widehat{\Phi}(\sigma)\right] \\
& \cdot\left[\mathbf{1}_{\Gamma_{k]}}(\sigma) \mathbf{1}_{\sigma}(k) \overline{\widehat{\Psi}(\sigma)}\right] \\
& =\sum_{k=0}^{\infty} \sum_{\sigma \in \Gamma} \lambda_{\sigma}^{-2 p} \widehat{\mathfrak{r}_{k} \mathfrak{a}_{k}^{\dagger} \mathfrak{a}_{k}} \Phi(\sigma) \widehat{\widehat{\mathfrak{F}_{k} \mathfrak{a}_{k}^{\dagger} \mathfrak{a}_{k}} \Psi(\sigma)} \\
& =\sum_{k=0}^{\infty}\left\langle\mathfrak{\xi}_{k} \mathfrak{a}_{k}^{\dagger} \mathfrak{a}_{k} \Phi, \mathfrak{F}_{k} \mathfrak{a}_{k}^{\dagger} \mathfrak{a}_{k} \Psi\right\rangle_{-p} .
\end{aligned}
$$

This completes the proof.

Theorem 35 sets up covariant identities for generalized functionals of $Z$. The next theorem then gives meaningful upper bounds to variants of generalized functionals of $Z$.

Theorem 36. Let $\Phi \in \mathcal{S}_{p}^{*}(Z)$ for some $p \geq 0$. Then, its $p$ variant $\operatorname{var}_{p}(\Phi)$ makes sense, and moreover

$$
\operatorname{var}_{p}(\Phi) \leq \sum_{k=0}^{\infty}\left\|\mathfrak{a}_{k}^{\dagger} \mathfrak{a}_{k} \Phi\right\|_{-p}^{2}
$$

Proof. By Theorems 16, 18, and 27, we know that $\mathfrak{F}_{k} \mathfrak{a}_{k}^{\dagger} \mathfrak{a}_{k} \Phi$ belongs to $\mathcal{S}_{p}^{*}(Z)$ and

$$
\left\|\mathfrak{F}_{k} \mathfrak{a}_{k}^{\dagger} \mathfrak{a}_{k} \Phi\right\|_{-p} \leq\left\|\mathfrak{a}_{k}^{\dagger} \mathfrak{a}_{k} \Phi\right\|_{-p}, \quad k \geq 0
$$

This together with (71) and (73) yields

$$
\operatorname{var}_{p}(\Phi)=\sum_{k=0}^{\infty}\left\|\mathfrak{F}_{k} \mathfrak{a}_{k}^{\dagger} \mathfrak{a}_{k} \Phi\right\|_{-p}^{2} \leq \sum_{k=0}^{\infty}\left\|\mathfrak{a}_{k}^{\dagger} \mathfrak{a}_{k} \Phi\right\|_{-p}^{2} .
$$

This completes the proof.

A sequence $u=\left(u_{k}\right)$ of generalized functionals in $\mathcal{S}^{*}(Z)$ is said to be $\left(\mathfrak{E}_{k}\right)$-predictable if

$$
u_{k}=\mathfrak{E}_{k-1} u_{k}, \quad k \geq 0
$$

It is said to be $\left(\mathfrak{a}_{k}^{\dagger}\right)$-integrable if the series $\sum_{k=0}^{\infty} \mathfrak{a}_{k}^{\dagger} u_{k}$ converges strongly in $\mathcal{S}^{*}(Z)$. In that case, we call $\sum_{k=0}^{\infty} \mathfrak{a}_{k}^{\dagger} u_{k}$ the generalized stochastic integral of $u$ with respect to $\left(\mathfrak{a}_{k}^{\dagger}\right)$ and write

$$
\mathfrak{I}(u)=\sum_{k=0}^{\infty} \mathfrak{a}_{k}^{\dagger} u_{k}
$$

Theorem 37. Let $\Phi \in \mathcal{S}^{*}(Z)$. Then, the sequence $u=$

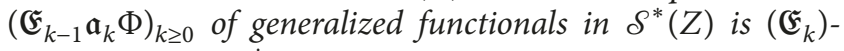
predictable and $\left(\mathfrak{a}_{k}^{\dagger}\right)$-integrable, and moreover

$$
\Phi=\mathfrak{E} \Phi+\mathfrak{I}(u) .
$$


Proof. This is an immediate consequence of Theorem 32.

Remark 38. A generalized functional of $Z$, or, in other words, a generalized functional in $\mathcal{S}^{*}(Z)$, can be interpreted as a generalized random variable on the probability space $(\Omega, \mathscr{F}, P)$. Accordingly, a sequence of generalized functionals of $Z$ can be viewed as a generalized stochastic process. Theorem 37 then shows that each generalized random variable on $(\Omega, \mathscr{F}, P)$ can be represented as the generalized stochastic integral of an $\left(\mathfrak{E}_{k}\right)$-predictable generalized stochastic process with respect to $\left(\mathfrak{a}_{k}^{\dagger}\right)$.

\section{Conflicts of Interest}

The authors declare that they have no conflicts of interest.

\section{Acknowledgments}

This work is supported by the National Natural Science Foundation of China (Grant no. 11461061).

\section{References}

[1] N. Privault, "Stochastic analysis of Bernoulli processes," Probability Surveys, vol. 5, pp. 435-483, 2008.

[2] C. Wang, Y. Lu, and H. Chai, "An alternative approach to Privault's discrete-time chaotic calculus," Journal of Mathematical Analysis and Applications, vol. 373, no. 2, pp. 643-654, 2011.

[3] F. Gao and N. Privault, "Clark formula and logarithmic Sobolev inequalities for Bernoulli measures," Comptes Rendus Mathematique, vol. 336, no. 1, pp. 51-56, 2003.

[4] C. Wang and J. Zhang, "Wick analysis for bernoulli noise functionals," Journal of Function Spaces, vol. 2014, Article ID 727341, 7 pages, 2014.

[5] I. M. Gel'fand and N. Y. Vilenkin, Generalized Functions, vol. 4, Academic Press, New York, NY, USA, 1964.

[6] C. Wang and J. Chen, "Characterization theorems for generalized functionals of discrete-time normal martingale," Journal of Function Spaces, vol. 2015, Article ID 714745, 6 pages, 2015.

[7] M. Émery, "A discrete approach to the chaotic representation property," in Séminaire de Probabilités XXXV, vol. 1755 of Lecture Notes in Mathematics, pp. 123-138, Springer, Berlin, Germany, 2001.

[8] J. J. Becnel, "Equivalence of topologies and Borel fields for countably-Hilbert spaces," Proceedings of the American Mathematical Society, vol. 134, no. 2, pp. 581-590, 2006.

[9] J. Muscat, Functional Analysis: An Introduction to Metric Spaces, Hilbert Spaces, and Banach Algebras, Springer International, Switzerland, 2014.

[10] C. Wang and J. Chen, "Convergence theorems for generalized functional sequences of discrete-time normal martingales," Journal of Function Spaces, vol. 2015, Article ID 360679, 7 pages, 2015. 


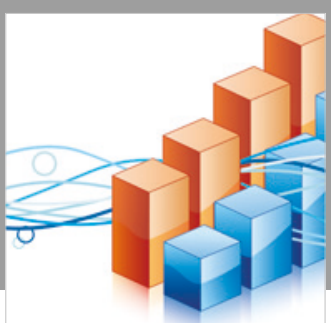

Advances in

Operations Research

\section{-n-m}
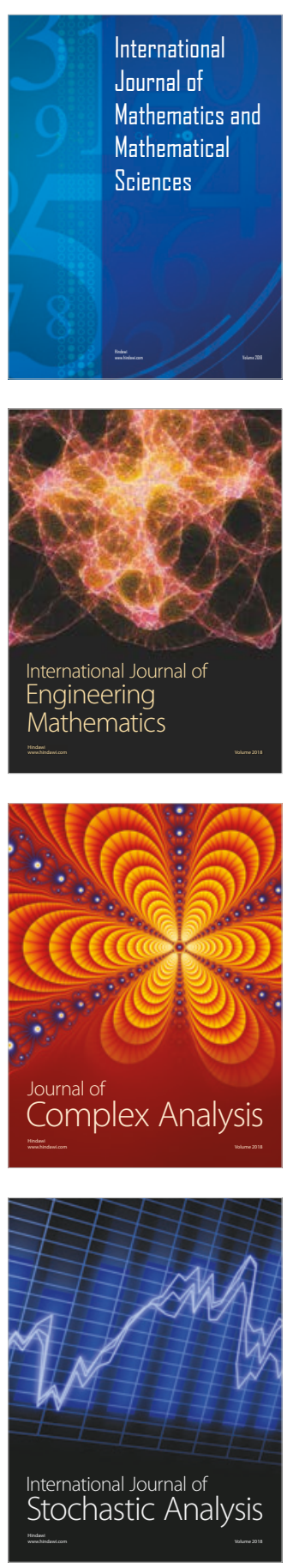
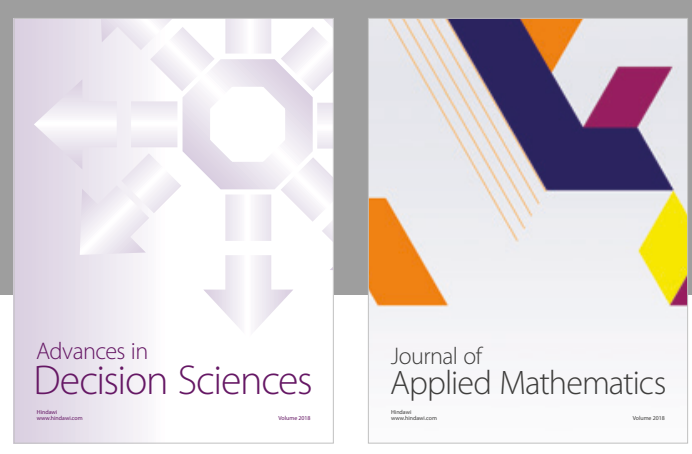

Journal of

Applied Mathematics
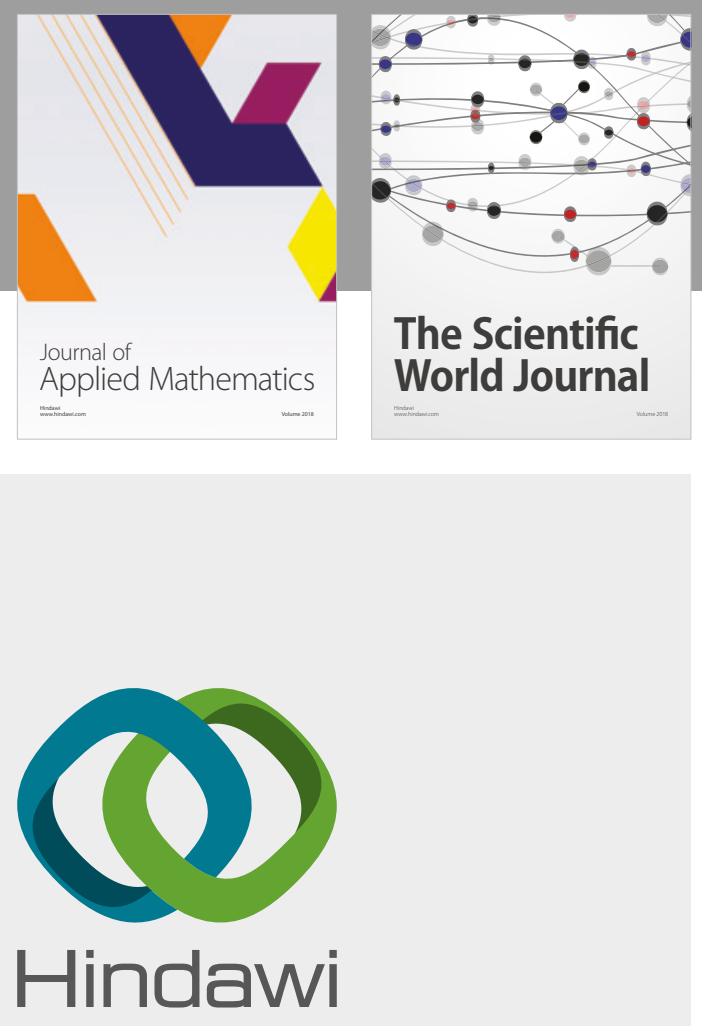

Submit your manuscripts at

www.hindawi.com

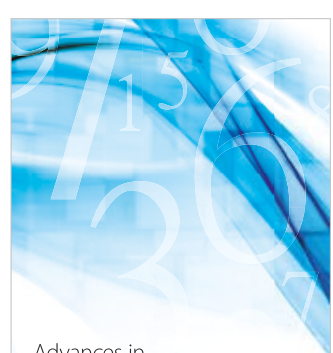

Advances in
Numerical Analysis
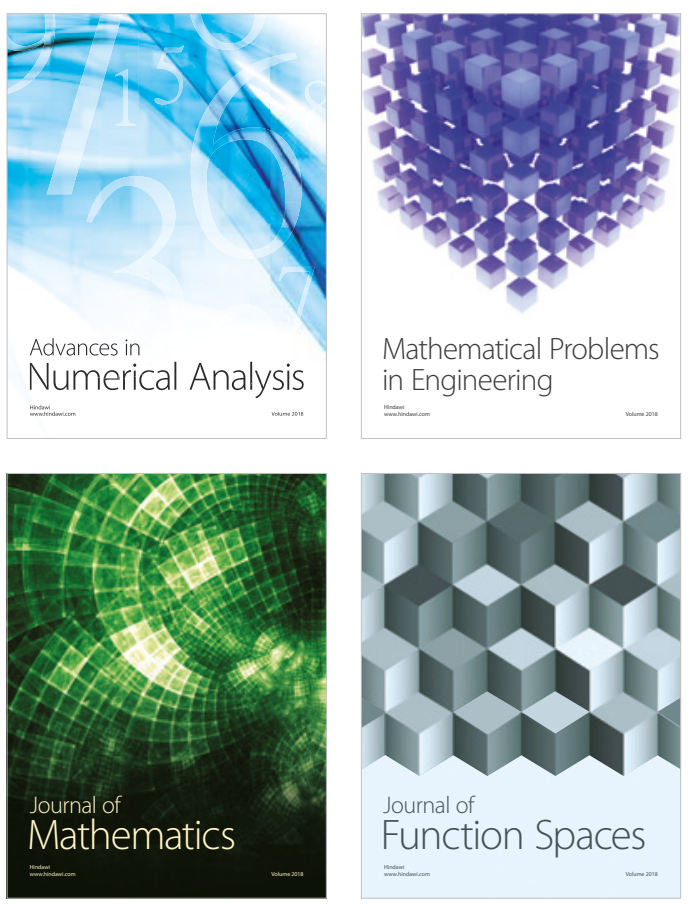

Mathematical Problems in Engineering

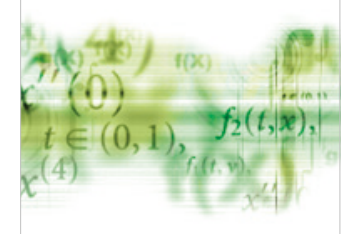

International Journal of

Differential Equations

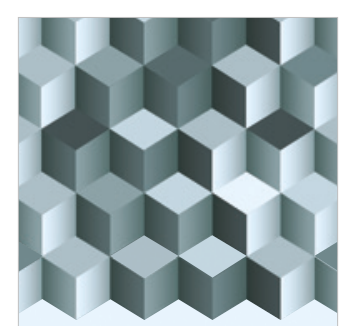

Journal of

Function Spaces

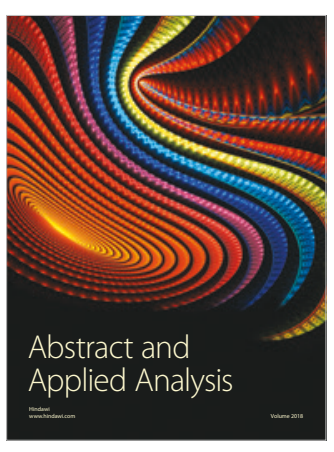

The Scientific

World Journal

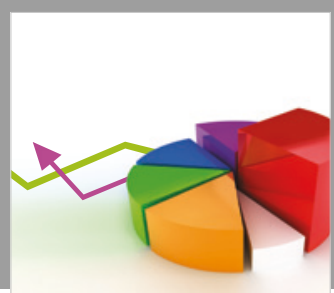

Journal of

Probability and Statistics
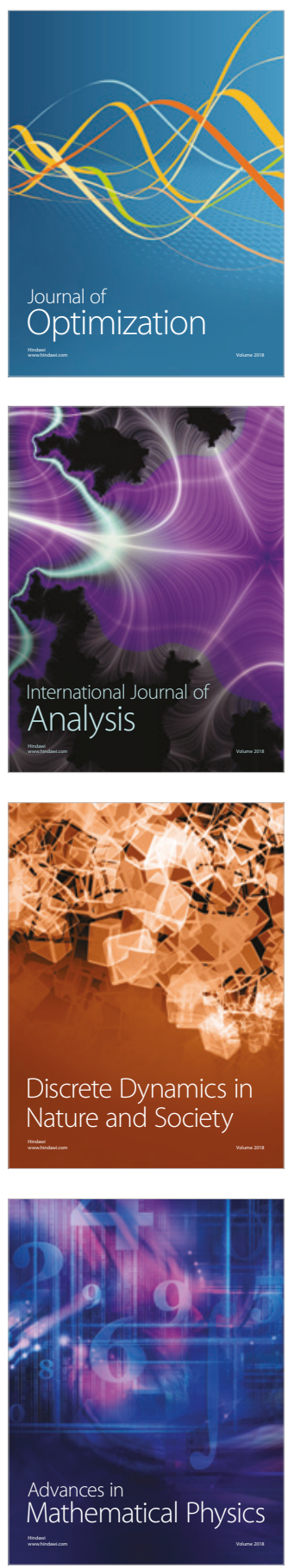